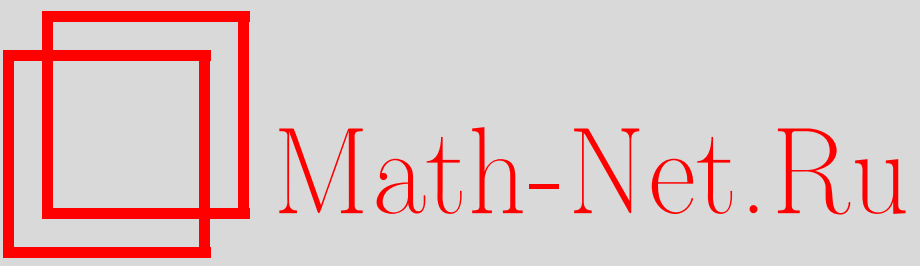

Г. Бахаа, Условия оптимальности систем с распределенными параметрами, использующие теорему Дубовицкого-Милютина с неполной информацией о начальных условиях, Итоги науки и техн. Сер. Соврем. мат. и ее прил. Темат. обз., 2020, том 178, 3-19

DOI: https://doi.org/10.36535/0233-6723-2020-178-3-19

Использование Общероссийского математического портала Math-Net.Ru подразумевает, что вы прочитали и согласны с пользовательским соглашением

http://www.mathnet.ru/rus/agreement

Параметры загрузки:

IP : 54.162 .85 .209

26 апреля 2023 г., 15:25:20 


\title{
УСЛОВИЯ ОПТИМАЛЬНОСТИ \\ СИСТЕМ С РАСПРЕДЕЛЕННЫМИ ПАРАМЕТРАМИ, ИСПОЛЬЗУЮЩИЕ ТЕОРЕМУ ДУБОВИЦКОГО-МИЛЮТИНА С НЕПОЛНОЙ ИНФОРМАЦИЕЙ О НАЧАЛЬНЫХ УСЛОВИЯХ
}

\author{
(c) 2020 г. $\quad$ Г. M. БAXAA
}

\begin{abstract}
АннотАция. Рассмотрена задача оптимального управления для системы, описанной с помощью линейного уравнения в частных производных параболического типа с граничными условиями второго рода. Зафиксированы некоторые ограничения на управления. Функционал качества имеет интегральную форму. Время управления $T$ зафиксировано. Начальное условие не определяется известной функцией, оно принадлежит некоторому множеству (неполная информация о начальном положении). Для получения условий оптимальности в задаче Неймана использовалось обобщение теоремы Дубовицкого-Милютина. Задача, сформулированная в данной статье, описывает процесс оптимального нагрева, для которого не известна точная информация о начальной температуре нагреваемого объекта. Также представлен пример, в котором допустимые управления и одно из начальных условий заданы с помощью ограничений на норму.
\end{abstract}

Ключевые слова: оптимальная задачв управления, задача Неймана, параболический оператор второго порядка, теорема Дубовицкого-Милютина, коническая аппроксимация, условия оптимальности.

\author{
OPTIMALITY CONDITIONS \\ FOR DISTRIBUTED PARAMETER SYSTEMS \\ USING DUBOVITSKII-MILYUTIN'S THEOREM \\ WITH INCOMPLETE INFORMATION \\ ABOUT THE INITIAL CONDITIONS
}

(C) 2020 G. M. BAHAA

\begin{abstract}
In this paper, we consider an optimal control problem for a system described by a linear partial differential equation of the parabolic type with Neumann's boundary condition. We impose some constraints on the control. The performance functional has the integral form. The control time $T$ is fixed. The initial condition is not given by a known function but belongs to a certain set (incomplete information about the initial state). To obtain optimality conditions for the Neumann problem, the generalization of the Dubovitskii-Milyutin theorem was applied. The problem formulating in this paper describes the process of optimal heating, of which we do not have exact information about the initial temperature on the heating object. We present an example in which the admissible controls and one of initial conditions are given by means of the norm constraints too.
\end{abstract}

Keywords and phrases: optimal control problem, Neumann problem, second-order parabolic operator, Dubovitskii-Milyutin theorem, conical approximations, optimality conditions.

AMS Subject Classification: 37N10, 76N15, 76U05 
1. Введение. Системы с распределенными параметрами можно использовать для описания многих феноменов в реальном мире. Известно, что теплопроводность, свойства эластичнопластичного материала, динамика жидкости, диффузионно-реакционные процессы, передача сигнала на некоторое расстояние с использованием длинных линий электропередачи и т.д., все они лежат внутри этой области. Изучаемый объект (температура, смещение, концентрация, скорость и т.п.) обычно называют состоянием.

Поэтому уравнения для систем с распределенными параметрами широко применяется в задачах оптимального управления, например, в $[12,13]$. В [20-22] для систем с распределенными параметрами обсуждаются задачи оптимального управления, которые задаются параболическими и гиперболическими операторами бесконечного порядка. Для получения условий оптимальности используются идеи работы [6].

В [14-17] применяется классическая теория управления для дифференциальных систем дробного порядка в ограниченной области. Рассматривается задача оптимального управления (ЗОУ) для дифференциальных систем дробного порядка (ДСДП) с запаздыванием и без. Рассматриваются дробные производные по времени в смысле Римана-Лиувилля и Капуто. Сначала изучается существование и единственность решения для ДСДП в гильбертовом пространстве. Затем показывается, что рассмотренная ЗОУ имеет единственное решение.

При помощи теоремы Дубовицкого-Милютина результатов работ $[8-11,18,23,24]$ в $[1,4,5,27$, 28] были получены необходимые и достаточные условия оптимальности для похожих систем, которые определяются оператором второго порядка с бесконечным числом переменных. Интерес в изучении этого класса операторов вызван задачами из квантовой теории поля.

Цель данной статьи - показать пользу метода Дубовицкого-Милютина в решении ЗОУ для систем с распределенными параметрами. Например, рассмотрена ЗОУ, описываемая линейным уравнением в частных производных параболического типа. Начальное условие для этого уравнения не определяется известной функцией, оно принадлежит некоторому множеству (начальное положение не задано априори). Правая часть этого уравнения и начальное условие обычно не являются непрерывными функциями, но являются измеримыми функциями из пространств $L^{2}$ либо $L^{\infty}$. Таким образом, решение этого уравнения задается некоторыми пространствами Соболева. Функционал качества имеет квадратичную форму, время управления фиксировано, на управления наложены ограничения. При помощи теоремы Дубовицкого-Милютина получены необходимые и достаточные условия оптимальности для задачи Неймана с квадратичным функционалом качества и ограниченным управлением.

Данная статья имеет следующую структуру. В разделе 2 введены определения пространств Соболева второго порядка, заданы некоторые определения конусов и сформулирована теорема Дубовицкого-Милютина. В разделе 3 введено параболическое уравнение для систем с распределенными параметрами. В разделе 4 сформулирована задача оптимального управления и приведены основные результаты работы. В заключительном разделе 5 подведены итоги.

2. Предварительные сведения. Цель данного раздела - дать некоторые начальные сведения и определения, необходимые в этой статье.

2.1. Соболевские пространства второго порядка. В этом разделе приводятся определения некоторых функциональных пространств второго порядка и цепочек составленных пространств, которые используются после.

Определение 2.1. Пусть $\Omega$ - ограниченное открытое множество в $\mathbb{R}^{n}$ с гладкой границей $\Gamma$, являющейся $C^{\infty}$-многообразием размерности $n-1$. Локально множество $\Omega$ полностью находится по одну сторону от Г. Пространство Соболева $L^{2}(\Omega)$ есть пространство квадратично интегрируемых функций $\phi(x)$, которое определяется на $\Omega$ следующим образом:

$$
L^{2}(\Omega)=\left\{\phi(x): \int_{\Omega}|\phi(x)|^{2} d x<\infty\right\} .
$$


Пространство $L^{2}(\Omega)$ гильбертово со скалярным произведением

$$
\langle\phi, \psi\rangle_{L^{2}(\Omega)}=\int_{\Omega} \phi(x) \psi(x) d x
$$

и нормой

$$
\|\phi\|_{L^{2}(\Omega)}=\left(\int_{\Omega}|\phi(x)|^{2} d x\right)^{1 / 2}=\sqrt{\langle\phi, \phi\rangle_{L^{2}(\Omega)}} .
$$

Определение 2.2. Пространство Соболева $H^{1}(\Omega)$ порядка 1 - это пространство

со скалярным произведением

$$
H^{1}(\Omega)=\left\{\phi \mid \phi \in L^{2}(\Omega), \frac{\partial \phi}{\partial x_{i}} \in L^{2}(\Omega), i=1,2, \ldots, n\right\}
$$

$$
\langle\phi, \psi\rangle_{H^{1}(\Omega)}=\langle\phi, \psi\rangle_{L^{2}(\Omega)}+\sum_{i=1}^{n}\left\langle\frac{\partial \phi}{\partial x_{i}}, \frac{\partial \psi}{\partial x_{i}}\right\rangle_{L^{2}(\Omega)},
$$

где $\partial \phi / \partial x_{i}$ - производные обобщенных функций. Пространство $H^{1}(\Omega)$, снабженное скалярным произведением (2.1), является гильбертовым пространством.

Определение 2.3. Пространство $H_{0}^{1}(\Omega) \subset H^{1}(\Omega)$ - это подпространство

со скалярным произведением (2.1).

$$
H_{0}^{1}(\Omega)=\left\{\phi\left|\phi \in H^{1}(\Omega), \phi\right|_{\Gamma}=0\right\}
$$

Обозначим через $X^{*}$ дуальное пространство $X$ и введем следующие пространства.

Определение 2.4. Пусть

$$
\left(H_{0}^{1}(\Omega)\right)^{*}=H_{0}^{-1}(\Omega), \quad\left(H^{1}(\Omega)\right)^{*}=H^{-1}(\Omega) .
$$

Имеем следующую цепочку включений:

$$
H_{0}^{1}(\Omega) \subseteq H^{1}(\Omega) \subseteq L^{2}(\Omega) \subseteq H_{0}^{-1}(\Omega), \quad H^{1}(\Omega) \subseteq L^{2}(\Omega) \subseteq H^{-1}(\Omega) .
$$

Определение 2.5. Введем пространство $L^{2}(Q) \equiv L^{2}\left(0, T ; L^{2}(\Omega)\right)$, где $\left.Q=\Omega \times\right] 0, T[$ - пространство таких измеримых функций $t \rightarrow \phi(t)$, что

$$
\|\phi\|_{L^{2}(Q)}=\left(\int_{0}^{T}\|\phi(t)\|_{2}^{2} d t\right)^{1 / 2}<\infty
$$

снабженное скалярным произведением

$$
(f, g)=\int_{0}^{T}(f(t), g(t))_{L^{2}(\Omega)} d t
$$

Пространство $L^{2}(Q)$ является гильбертовым пространством.

Аналогично определим пространства $L^{2}\left(0, T ; H_{0}^{1}(\Omega)\right)$ и $L^{2}\left(0, T ; H^{1}(\Omega)\right)$, а также $L^{2}\left(0, T ; H_{0}^{-1}(\Omega)\right)$ и $L^{2}\left(0, T ; H^{-1}(\Omega)\right)$ как их формальные сопряженные.

Имеет место следующая цепочка включений:

$$
\begin{aligned}
& L^{2}\left(0, T ; H_{0}^{1}(\Omega)\right) \subseteq L^{2}(Q) \subseteq L^{2}\left(0, T ; H_{0}^{-1}(\Omega)\right), \\
& L^{2}\left(0, T ; H^{1}(\Omega)\right) \subseteq L^{2}(Q) \subseteq L^{2}\left(0, T ; H^{-1}(\Omega)\right) .
\end{aligned}
$$

Введем также пространство

$$
W(0, T):=\left\{y \mid y \in L^{2}\left(0, T ; H^{1}(\Omega)\right), \frac{\partial y}{\partial t} \in L^{2}\left(0, T ; H^{-1}(\Omega)\right)\right\},
$$


в котором будет содержаться решение параболического уравнения. Рассматриваемые в данной работе пространства считаются действительными.

2.2. Определения конусов и теорема отделимости. Метод Дубовицкого-Милютина (см. [4,5]) возникает из теоремы Хана-Банаха (теорема о разделении выпуклых конусов). Это будет показано в примере ниже.

Предположим, что $E$-линейное локально выпуклое топологическое пространство, $I(x)-$ функционал, определенный на $E, A_{i}, i=1,2, \ldots, n$, - множества в $E$ с внутренними точками, которые представляют собой ограничения-неравенства, $B$-множество в $E$ без внутренних точек, представляющее ограничения-равенства.

Необходимо найти некоторые условия, необходимые для локального минимума функционала $I(x)$ на множестве $Q=\bigcap_{i=1}^{n} A_{i} \cap B$, либо найти такую точку $x_{0} \in E$, что $I\left(x_{0}\right)=\min _{Q \cup U} I(x)$, где $U-$ некоторая окрестность точки $x_{0}$. Определим множество

$$
A_{0}=\left\{x: I(x)<I\left(x_{0}\right)\right\} .
$$

Далее сформулируем необходимое условие оптимальности следующим образом: в окрестности точки локального минимума пересечение класса множеств (множество, в котором функционал достигает значений, меньших, чем $I\left(x_{0}\right)$, а множества представляют ограничения) либо является пустым, либо $\bigcap_{i=0}^{n} A_{i} \cap B=\phi$.

Условие $\bigcap_{i=0}^{n} A_{i} \cap B=\phi$ также эквивалентно условию, в котором вместо множеств $A_{i}, i=$ $1,2, \ldots, n$, и $B$ используются их аппроксимации. Эти аппроксимации суть конусы с вершиной в точке $\{0\}$.

Аппроксимируем ограничения в виде неравенств с помощью регулярных допустимых конусов $R A C\left(A_{i}, x_{0}\right), i=1,2, \ldots, n$, ограничения в виде равенства - с помощью регулярного касательного конуса $R T C\left(B, x_{0}\right)$, а для функционала качества построим регулярное улучшение конуca $\operatorname{RFC}\left(I, x_{0}\right)$. Таким образом, необходимое условие оптимальности $I(x)$ на множестве $Q=\bigcap_{i=1}^{n} A_{i} \cap B$ прини-
мает форму уравнения Эйлера-Лагранжа

$$
\sum_{i=0}^{n+1} f_{i}=0,
$$

где $f_{i}(i=0,1, \ldots, n+1)$ - линейные непрерывные функционалы; все они не обращаются в нуль в один и тот же момент времени и принадлежат сопряженным конусам

$$
\begin{gathered}
f_{i} \in\left[R A C\left(A_{i}, x_{0}\right)\right]^{*}, i=1,2, \ldots, n, \quad f_{n+1} \in\left[R T C\left(B, x_{0}\right)\right]^{*}, \quad f_{0} \in\left[R F C\left(I_{i}, x_{0}\right)\right]^{*}, \\
f_{i} \in\left[R A C\left(A_{i}, x_{0}\right)\right]^{*} \Longleftrightarrow f_{i}(x) \geqslant 0 \forall x \in R A C\left(A_{i}, x_{0}\right), i=1,2, \ldots, n .
\end{gathered}
$$

Для выпуклых задач, т.е. задач, в которых ограничения являются выпуклыми множествами и функционал качества тоже выпуклый, уравнение Эйлера-Лагранжа есть необходимое и достаточное условие оптимальности при условии, что выполнены определенные дополнительные предположения (так называемое условие Слейтера).

Используя теорему Милютина-Дубовицкого, выведем необходимые и достаточные условия оптимальности для параболических систем с неполной информацией о начальном состоянии.

Напомним сначала определения конических аппроксимаций и сонаправленных или противоположно направленных конусов. Пусть $A$ - множество, содержащееся в банаховом пространстве $X$, и $F: X \rightarrow \mathbb{R}$ - заданный функционал.

Определение 2.6. Множество

$$
T C\left(A, x^{0}\right):=\left\{h \in X \mid \exists \epsilon_{0}>0 \forall \epsilon \in\left(0, \epsilon_{0}\right) \exists r(\epsilon) \in X x^{0}+\epsilon h+r(\epsilon) \in A\right\},
$$

где $r(\epsilon) / \epsilon \rightarrow 0$ при $\epsilon \rightarrow 0$, называется касательным конусом к множеству $A$ в точке $x^{0} \in A$. 
Определение 2.7. Множество

$$
A C\left(A, x^{0}\right):=\left\{h \in X \mid \exists \epsilon_{0}>0 \exists U(h) \forall \epsilon \in\left(0, \epsilon_{0}\right) \forall \bar{h} \in U(h) x^{0}+\epsilon \bar{h} \in A\right\},
$$

где $U(h)$ - окрестность $h$, называется допустимым конусом к множеству $A$ в точке $x^{0} \in A$.

Определение 2.8. Множество

$$
F C\left(F, x^{0}\right):=\left\{h \in X \mid \exists \epsilon_{0}>0 \exists U(h) \forall \epsilon \in\left(0, \epsilon_{0}\right) \forall \bar{h} \in U(h) F\left(x^{0}+\epsilon \bar{h}\right)<F\left(x^{0}\right)\right\}
$$

называется конусом уменьшения функционала $F$ в точке $x^{0} \in X$.

Определение 2.9. Множество

$$
N C\left(F, x^{0}\right):=\left\{h \in X \mid \exists \epsilon_{0}>0 \exists U(h) \forall \epsilon \in\left(0, \epsilon_{0}\right) \forall \bar{h} \in U(h) F\left(x^{0}+\epsilon \bar{h}\right) \leqslant F\left(x^{0}\right)\right\}
$$

называется конусом неувеличения функционала $F$ в точке $x^{0} \in X$.

Все определенные выше конусы являются конусами с вершиной в начале координат. Конусы $A C\left(A, x^{0}\right), F C\left(F, x^{0}\right)$ и $N C\left(F, x^{0}\right)$ являются открытыми, а конус $T C\left(A, x^{0}\right)$ - замкнутым. Если $\operatorname{int} A \neq \varnothing$, то $A C\left(A, x^{0}\right)$ не существует. Более того, если $A_{1}, \ldots, A_{n} \in X, x^{0} \in \bigcap_{i=1}^{n} A_{i}$, то

$$
\bigcap_{i=1}^{n} T C\left(A_{i}, x^{0}\right) \supset T C\left(\bigcap_{i=1}^{n} A_{i}, x^{0}\right), \quad \bigcap_{i=1}^{n} A C\left(A_{i}, x^{0}\right)=A C\left(\bigcap_{i=1}^{n} A_{i}, x^{0}\right) .
$$

Если конусы $T C\left(A, x^{0}\right), A C\left(A, x^{0}\right), F C\left(F, x^{0}\right)$ и $N C\left(F, x^{0}\right)$ выпуклы, то они называются регулярными конусами и обозначаются соответственно $R T C\left(A, x^{0}\right), R A C\left(A, x^{0}\right), R F C\left(F, x^{0}\right)$ и $\operatorname{RNC}\left(F, x^{0}\right)$.

Пусть $C_{i}, i=1, \ldots, n,-$ система конусов, $B_{M}$-шар с центром в 0 и радиусом $M>0$ в пространстве $X$.

Определение 2.10. Конусы $C_{i}, i=1, \ldots, n$, называются сонаправленными, если для любого $M>0$ существуют такие $M_{1}, \ldots, M_{2}>0$, что при всех $x \in B_{M} \cap \sum_{i=1}^{n} C_{i}$ вида $x=\sum_{i=1}^{n} x_{i}$, где $x_{i} \in C_{i}$, $i=1, \ldots, n$, имеем $x_{i} \in B_{M_{i}} \cap C_{i}, i=1, \ldots, n$, или, эквивалентно, неравенство $\|x\| \leqslant M$ влечет неравенства $\left\|x_{i}\right\| \leqslant M_{i}, i=1, \ldots, n$.

Определение 2.11. Будем говорить, что конусы $C_{i}, i=1, \ldots, n$, имеют противоположное направление, если найдется такой $\left(x_{1}, \ldots, x_{n}\right) \neq(0, \ldots, 0), x_{i} \in C_{i}, i=1, \ldots, n$, что $\sum_{i=1}^{n} x_{i}=0$.

Замечание 2.1. Из определений 2.5 и 2.6 следует, что множество сонаправленных конусов не пересекается с множеством конусов противоположного направления. Если некоторая подсистема конусов имеет противоположное направление, то и вся система также противоположно направлена.

В конечномерных пространствах существуют только конусы двух типов, упомянутые выше (см. [29]), тогда как в произвольных бесконечномерных нормированных пространствах ситуация более сложна, как показывает следующий пример.

Пример 2.1. В пространстве $C^{1}[0,1]$ с нормой

$$
\|x\|:=\left(\int_{0}^{1} x^{2}(t) d t\right)^{1 / 2}
$$

определим функционал

$$
A(x):=\left.\frac{d}{d t} x(t)\right|_{t=1 / 2}=: r \in \mathbb{R}
$$


Этот функционал линеен и ограничен. Для последовательности $x_{n}(t)=\sin 2 \pi n t / \sqrt{n}$ имеем $\left\|x_{n}\right\|=1 / \sqrt{2 n} \rightarrow 0$, однако $\left.A\left(x_{n}\right)=2 \pi \sqrt{n} \cos \pi n=2 \pi \sqrt{n}(-1)^{n} \not \rightarrow 0\right)$. Далее введем замкнутые и выпуклые конусы $C_{1}:=\operatorname{cl}\{(x, r) ; r=A(x)\}$ и $C_{2}:=\{\Theta\} \times \mathbb{R}$ в пространстве $E:=L^{2}(0,1) \times \mathbb{R}$ с нормой $\|(x, r)\|_{E}:=\max \{\|x\|,|r|\}$. Конусы $C_{1}$ и $C_{2}$ направлены противоположно. Для того, чтобы это доказать, возьмём $v_{1}=\left(x_{1}, r_{1}\right) \in C_{1}, v_{2}=\left(\Theta, r_{2}\right) \in C_{2}$ и произвольную константу $M>0$. Если

$$
\left\|v_{1}+v_{2}\right\|_{E}=\max \left\{\left\|x_{1}\right\|,\left|r_{1}+r_{2}\right|\right\} \leqslant M,
$$

то неравенства

$$
\left\|v_{1}\right\|_{E} \leqslant M_{1}, \quad\left\|v_{2}\right\|_{E} \leqslant M_{2}
$$

не выполняются в общем случае с некоторыми $M_{1}, M_{2}>0$. На самом деле, так как $A$ - неограниченный функционал, то существует такое $\tilde{M}_{1}>0$, что $\left|r_{1}\right|=\left|A\left(x_{1}\right)\right|>\tilde{M}_{1}$. Далее зафиксируем $M<\tilde{M}_{1}$. Тогда

$$
M \geqslant\left|r_{1}+r_{2}\right| \geqslant\left|r_{1}\right|-\left|r_{2}\right|>\tilde{M}_{1}-\left|r_{2}\right|, \quad\left\|v_{2}\right\|_{E}=\tilde{M}_{1}-M=: M_{2}>0,
$$

что противоречит противоположной направленности конусов $C_{1}$ и $C_{2}$.

В [28] представлено условие, при котором система конусов является сонаправленной.

Определение 2.12. Пусть $K$-конус в $X$. Сопряженный конус $K^{*}$ для $K$ определим следующим образом:

$$
K^{*}:=\left\{f \in X^{*} ; f(x) \geqslant 0 \forall x \in K\right\},
$$

где $X^{*}$ - пространство, двойственное к $X$.

Определение 2.13. Пусть $Q$ - множество в $X, x^{0} \in Q$. Функционал $f \in X^{*}$ называется опорным функционалом для множества $Q$ в точке $x^{0}$, если $f(x) \geqslant f\left(x^{0}\right)$ для всех $x \in Q$.

Сформулируем теорему о разделении выпуклых конусов.

Теорема 2.1. Предположим, что выполнены следующие условия:

(i) $K_{1}, \ldots, k_{p} \subset X$-открытые и выпуклые конусы с верииной в 0 ,

(ii) $K_{p+1}, \ldots, K_{n} \subset X, n>p$, - замкнутые и выпуклые конусы с вершиной в 0 ,

(iii) сопря⿻енные конусы $K_{p+1}^{*}, \ldots, K_{n}^{*} \partial л я K_{p+1}, \ldots, K_{n}$ соответственно являются либо сонаправленными, либо противоположно направленнымию

Тогда равенство

$$
\bigcap_{i=1}^{n} K_{i}=\varnothing
$$

имеет место в том и только том случае, когда существуют такие линейные непрерывные функциональ $f_{i} \in K_{i}^{*}, i=1, \ldots, n$, не обращающиеся одновременно в нуль, что выполняется соотношение

$$
\sum_{i=1}^{n} f_{i}=0
$$

называемое уравнением Эйлера-Лагранжа.

Доказательство. Достаточность. Рассмотрим только случай, когда конусы $K_{p+1}^{*}, \ldots, K_{n}^{*}$ сонаправлены, так как случай, когда конусы противоположно направлены, тривиален (см. замечание 2.1). Введем обозначения

$$
K:=\bigcap_{i=1}^{p} K_{i}, \quad C:=\bigcap_{i=p+1}^{n} K_{i} .
$$

Возможны четыре случая:
(1) $K \neq \varnothing, C \neq \varnothing$;
(2) $K=\varnothing, C \neq \varnothing$;
(3) $K \neq \varnothing, C=\varnothing$;
(4) $K=\varnothing, C=\varnothing$. 
В первом случае $K$ и $C$-открытые выпуклые конусы с вершиной в 0 . Конусы $K$ и $C$ можно разделить (см. [29, лемма 5.11]). Это означает, что существует такой ненулевой линейный непрерывный функционал $\varphi$, что

$$
\varphi \in\left(\bigcap_{i=1}^{p} K_{i}\right)^{*}, \quad-\varphi \in\left(\bigcap_{i=p+1}^{n} K_{i}\right)^{*} .
$$

Используя лемму 5.11 из [1], имеем следующее представление для $\varphi$ :

$$
\varphi=\sum_{i=1}^{p} f_{i}, \quad \text { где } \quad f_{i} \in K_{i}^{*}, i=1, \ldots, p .
$$

Из [1, лемма 5.10] и $[29$, теорема 3.5$]$ получаем

$$
\left(\bigcap_{i=p+1}^{n} K_{i}\right)^{*}=\overline{\operatorname{conv} \sum_{i=p+1}^{n} K_{i}^{*}}=\overline{\sum_{i=p+1}^{n} K_{i}^{*}}=\sum_{i=p+1}^{n} K_{i}^{*}
$$

где верхняя черта обозначает замыкание в слабой $*$-топологии. Следовательно, $-\varphi$ имеет следующее представление:

$$
-\varphi=\sum_{i=p+1}^{n} f_{i}, \quad \text { где } \quad f_{i} \in K_{i}^{*}, i=p+1, \ldots, n .
$$

Принимая во внимание $(2.1),(2.2)$, получаем $\sum_{i=1}^{n} f_{i}=0$, что завершает доказательство теоремы в первом случае.

Для второго случая имеем $\bigcap_{i=1}^{p} K_{i}=\varnothing$, откуда следует, что существует такое $s, 1 \leqslant s<p$, что $\bigcap_{i=1}^{s} K_{i} \neq \varnothing$ и $\bigcap_{i=1}^{s+1} K_{i}=\varnothing$. Продолжая аналогично случаю $(1)$, получаем уравнение ЭйлераЛагранжа с $f_{s+2}=\cdots=f_{n}=0$. Случаи (3) и (4) анализируются аналогично.

Необходимость. Пусть $\sum_{i=1}^{n} f_{i}=0$ и $\bigcap_{i=1}^{n} k_{i} \neq \varnothing$. Тогда существует $x^{0} \in K_{i}, i=1, \ldots, n$. Для некоторого $j, 1 \leqslant j \leqslant p$, выполнено $f_{j}^{i=1} \neq 0$ (поскольку $\varphi \neq 0$ ). Так как $K_{j}$-открытый конус, то $f_{j}\left(x^{0}\right)>0$. Отсюда $0=\sum_{i=1}^{n} f_{i}\left(x^{0}\right) \geqslant f_{j}\left(x^{0}\right)>0$, что невозможно.

2.3. Постановка оптимальных задач. Пусть $X$-банахово пространство, $Q_{k} \subset X$, int $Q_{k} \neq \varnothing$, $k=1, \ldots, p$ задает ограничения-неравенства, $Q_{k} \subset X$, int $Q_{k}=\varnothing, k=p+1, \ldots, n$, определяет ограничения-равенства, $I_{i}:=X \rightarrow \mathbb{R}, i=1, \ldots, s$, задается функционалами $I=\left(I_{1}, \ldots, I_{s}\right)^{T}$, т.е. $I: X \rightarrow \mathbb{R}^{s}$ - векторный функционал качества. Интерес представляет следующая задача (см. [1]). Задача $P$. Найти такой $x^{0} \in Q$, что $\min _{x \in Q \cap U\left(x^{0}\right)} I(x)=I\left(x^{0}\right)$, где $Q=\bigcap_{k=1}^{n} Q_{k}$ и $U\left(x^{0}\right)$ - некоторая
окрестность $x^{0}$.

Если определить ограничения-равенства в форме оператора

$$
Q_{k}:=\left\{x \in X: F_{k}(x)=0\right\}
$$

где $F_{k}: X \rightarrow Y_{k}$ - заданные операторы, $Y_{k}$ - банаховы пространства, $k=p+1, \ldots, n$, то соответствующую задачу будем называть задачей $P 1$.

Определение 2.14. Точка $x^{0} \in X$ называется глобально (локально) оптимальной для задачи $P$ или $P 1$, если $x^{0} \in Q$ и не существует $x \in Q, x \neq x^{0}\left(Q \cap U\left(x^{0}\right)\right)$, удовлетворяющего условию $I_{i}(x) \leqslant I\left(x^{0}\right), i=1, \ldots, s$, со строгим неравенством для по крайней мере одного $i$ из диапазона $1 \leqslant i \leqslant s$.

Теорема 2.2 (обобщенная теорема Дубовицкого-Милютина; см. [23]). Предположим, что для задачи $(P)$ выполнены следующие условия: 
(i) конусы $K_{i}, i=1, \ldots, s, D_{j}, j=1, \ldots, s, C_{k}, k=1, \ldots, p$, открыты и выпуклы;

(ii) конусы $C_{k}, k=p+1, \ldots, n$, выпуклы и замкнуты;

(iii) конус $\tilde{C}=\bigcap_{k=p+1}^{n} C_{k}$ содержится в конусе, касательном кмножеству $\bigcap_{k=p+1}^{n} Q_{k}$;

(iv) конусы $C_{k}^{*}, k=p+1, \ldots, n$, являются либо сонаправленными, либо противоположсно направленными;

(v) $x^{0} \in Q$ есть локальный оптимум для задачи $(P)$.

Тогда выполняются следующие s уравнений (так называемые уравнения Эйлера- Лагрнљса):

$$
f_{i}+\sum_{\substack{j=1, j \neq i}}^{s} f_{j}^{(i)}+\sum_{k=1}^{n} \varphi_{k}^{(i)}=0, \quad i=1,2, \ldots, s,
$$

где $f_{i} \in K_{i}^{*}, f_{j}^{(i)} \in D_{j}^{*}, j=1, \ldots, s, j \neq i, \varphi_{k}^{(i)} \in C_{k}^{*}, k=1, \ldots, n$, с функционалами, не обращающимися в нуль одновременно.

Случай $s=1$. При $s=1$ задачи $(P)$ и $(P 1)$ становятся скалярными задачами, для которых справедлива следующая теорема.

Теорема 2.3 (см. [23]). Допустим, что для задачи (P) выполнены следующие условия:

(i) $s=1$;

(ii) $I: X \rightarrow \mathbb{R}$ выпукло и непрерывно;

(iii) $Q_{k}, k=1, \ldots, n$ выпукль;

(iv) существует такое $\tilde{x}$, что

$$
\tilde{x} \in\left(\bigcap_{k=1}^{p} \operatorname{int} Q_{k}\right) \cap\left(\bigcap_{k=p+1}^{n} \operatorname{int} Q_{k}\right) ;
$$

(v) выполняется равенство

$$
\operatorname{RTC}\left(\bigcap_{k=p+1}^{n} Q_{k}, x^{0}\right)=\bigcap_{k=p+1}^{n} R T C\left(Q_{k}, x^{0}\right) ;
$$

(vi) конусы $\left[R T C\left(Q_{k}, x^{0}\right)\right]^{*}, k=p+1, \ldots, n$, являются сонаправленными либо противоположно направленными.

Точка $x^{0}$ является глобальным оптимумом для задачи $(P)$ тогда и только тогда, когда выполнено уравнение Эйлера-Лагранюса

$$
f_{1}+\sum_{k=1}^{n} \varphi_{k}=0
$$

где $f \in\left[R F C\left(I, x^{0}\right)\right]^{*}, \varphi_{k} \in\left[R A C\left(Q_{k}, x^{0}\right)\right]^{*}, k=1, \ldots, n$, и функциональ не обращалотся в нуль одновременно.

Используя теорему Дубовицкого-Милютина, выведем необходимые и достаточные условия оптимальности для параболических систем с неполной информацией о начальном состоянии.

3. Постановка задачи оптимального управления и теорема оптимизации. Цель данного раздела - сформулировать следующие смешанные начальные граничные значения для задачи Неймана с параболической системой, которая определяет следующую модель:

$$
\begin{array}{ll}
\frac{\partial y}{\partial t}+\mathcal{A}(t) y=u, & x \in \Omega, t \in(0, T) ; \\
\frac{\partial y}{\partial \nu_{\mathcal{A}}}=0, & x \in \Gamma, t \in(0, T) ; \\
y(x, 0) \in K, & x \in \Omega,
\end{array}
$$


где $\Omega$ имеет те же свойства, что и в разделе 2 . Будем использовать обозначения

$$
y \equiv y(x, t), \quad u \equiv u(x, t), \quad Q=\Omega \times(0, T), \quad \bar{Q}=\bar{\Omega} \times[0, T], \Sigma=\Gamma \times(0, T),
$$

где $T$ - заданное положительное число (конечный промежуток времени), $K$ - замкнутое выпуклое подмножество с непустой внутренней частью в пространстве $H^{1}(\Omega), y$ - функция, определенная на $Q$, так что $\Omega \times(0, T) \ni(x, t) \rightarrow y(x, t) \in R$; наконец, $u$-такие функции, определенные на $Q$, что $\Omega \times(0, T) \ni(x, t) \rightarrow u(x, t) \in R$.

Оператор $\partial / \partial t+\mathcal{A}$ в уравнении состояния (3.1) является параболическим; оператор $\mathcal{A}$ задан следующим образом:

$$
\mathcal{A}(t) y=-\sum_{i, j=1}^{n} \frac{\partial}{\partial x_{i}}\left(a_{i j}(x, t) \frac{\partial y(x, t)}{\partial x_{j}}\right)+a_{0}(x, t) y(x, t),
$$

где $a_{i j}, i, j=1,2, \ldots, n$, - заданные вещественные функции на $\Omega$, удовлетворяющие условиям $a_{0}(x, t), a_{i j}(x, t) \in L^{\infty}(\Omega), a_{0}(x, t) \geqslant \alpha>0$ и

$$
\sum_{i, j=1}^{n} a_{i j}(x, t) \xi_{i} \xi_{j} \geqslant \alpha\left(\xi_{1}^{2}+\cdots+\xi_{n}^{2}\right)
$$

для всех $\xi \in \mathbb{R}^{n}$ почти всюду на $\Omega$. Можно проверить, что $\mathcal{A} \in \mathcal{L}\left(\left(H^{1}(\Omega), H^{-1}(\Omega)\right)\right.$.

Определение 3.1. Для каждого $t \in(0, T)$ определим семейство билинейных форм на $H^{1}(\Omega)$ следующим образом:

$$
\pi(t ; y, \phi)=(\mathcal{A} y, \phi)_{L^{2}(\Omega)}, \quad y, \phi \in H^{1}(\Omega),
$$

где $\mathcal{A}: H^{1}(\Omega) \rightarrow H^{-1}(\Omega)$. Тогда

$$
\begin{aligned}
& \pi(t ; y, \phi)=(\mathcal{A} y, \phi)_{L^{2}(\Omega)}=\left(-\sum_{i, j=1}^{n} \frac{\partial}{\partial x_{i}}\right.\left.\left(a_{i j}(x, t) \frac{\partial y(x, t)}{\partial x_{j}}\right)+a_{0}(x, t) y(x, t), \phi\right)_{L^{2}(\Omega)}= \\
&=\int_{\Omega} \sum_{i, j=1}^{n} a_{i j}(x, t) \frac{\partial y(x, t)}{\partial x_{j}} \frac{\partial \phi}{\partial x_{i}} d x+\int_{\Omega} a_{0}(x, t) y(x, t) \phi d x .
\end{aligned}
$$

Замечание 3.1. Билинейная форма $\pi(t ; y, \phi)$ является коэрцитивной на $H^{1}(\Omega)$, т.е.

$$
\pi(t ; y, y) \geqslant \alpha\|y\|_{H^{1}(\Omega)}^{2}
$$

для всех $y \in H^{1}(\Omega)$, где $\alpha>0$. Действительно, хорошо известно, что эллиптичность оператора $\mathcal{A}(t)$ является достаточной для коэрцитивности формы $\pi(t ; y, \phi)$ на $H^{1}(\Omega)$. Так как

$$
\pi(t ; y, \phi)=\int_{\Omega} \sum_{i, j=1}^{n} a_{i j}(x, t) \frac{\partial y(x, t)}{\partial x_{j}} \frac{\partial \phi}{\partial x_{i}} d x+\int_{\Omega} a_{0}(x, t) y(x, t) \phi d x,
$$

воспользуемся (3.5):

$$
\begin{gathered}
\pi(t ; y, y)=\int_{\Omega} \sum_{i, j=1}^{n} a_{i j}(x, t) \frac{\partial y}{\partial x_{j}} \frac{\partial y}{\partial x_{i}} d x+\int_{\Omega} a_{0}(x, t)|y|^{2} d x \geqslant \\
\geqslant \alpha \sum_{i, j=1}^{n}\left(\left\|\frac{\partial y}{\partial x_{i}}\right\|^{2}+\|y\|^{2}\right) \geqslant \alpha\|y\|_{H^{1}(\Omega)}^{2}, \quad \alpha>0 .
\end{gathered}
$$

Допустим также, что для всех $y, \phi \in H^{1}(\Omega)$ функция $t \rightarrow \pi(t ; y, \phi)$ является непрерывно дифференцируемой на $[0, T]$ и $\pi(t ; y, \phi)=\pi(t ; \phi, y)$. Уравнения (3.1)-(3.3) составляют задачу Неймана. Тогда левая часть граничного условия (3.2) может быть записана в следующей форме:

$$
\frac{\partial y(u)}{\partial \nu_{\mathcal{A}}}=\sum_{i=0}^{n} \frac{\partial}{\partial x_{i}} y(u) \cos \left(n, x_{k}\right)=0, \quad x \in \Gamma, t \in(0, T),
$$


где $\partial / \partial \nu_{\mathcal{A}}-$ производная по нормали к $\Gamma$, направленной внутрь $\Omega, \operatorname{a} \cos \left(n, x_{k}\right)-k$-й направляющий косинус с внешней нормалью $n$ к поверхности $\Gamma$.

Правая часть уравнения (3.1) и начальное условие (3.3) не являются непрерывными функциями, но являются измеримыми функциями, принадлежащими $L^{2}$ либо $L^{\infty}$; кроме того, начальное условие не задается известной функцией, но принадлежит некоторому множеству $K$ (начальное состояние не задано априори). Таким образом, будем искать решение уравнений (3.1)-(3.3) в некоторых соболевских пространствах.

Сформулируем достаточные условия для существования единственного решения смешанной начально-краевой задачи (3.1)-(3.3) для случая, где управление $u \in L^{2}(Q)$.

Следующая теорема может быть доказана с помощью [6, теорема 1.2].

Теорема 3.1. Если начальное состояние $y(x, 0)$ - произвольная фиксированная функиия $y(x, 0) \in K \subset H^{1}(\Omega)$ u $u \in L^{2}(Q)$, то существует единственное решение $y \in W(0, T)$ начальнокраевой задачи (3.1)-(3.3).

4. Формулировка задачи и теоремы оптимизации. Теперь сформулируем задачу оптимального управления для (3.1)-(3.3) в контексте теоремы 3.1 , а именно, $u \in L^{2}(Q)$.

Пусть $U=L^{2}(Q)$ - пространство управлений, $Y=L^{2}\left(0, T, H^{1}(\Omega)\right)$ - пространство состояний; время $T$ в нашей задаче фиксировано. Рассмотрим функционал качества

$$
I(y, u)=\lambda_{1} \int_{Q}\left[y(x, t ; u)-z_{d}\right]^{2} d x d t+\lambda_{2} \int_{Q}(N u) u d x d t
$$

где $\lambda_{i} \geqslant 0$ и $\lambda_{1}+\lambda_{2}>0, z_{d}$ - заданный элемент в $L^{2}(Q), N$ - строго положительный линейный оператор из $L^{2}(Q)$ в $L^{2}(Q)$.

Введем следующие ограничения на управления. Пусть $U_{a d}$ - множество допустимых управлений, определенных следующим образом:

$$
U_{a d} \text { - замкнутое выпуклое подмножество пространства } U=L^{2}(Q)
$$

$$
\text { с непустым внутренним подмножеством. }
$$

Пусть $y(x, t ; u)$ - решение смешанной начально-краевой задачи (3.1)-(3.3), соответствующее заданному управлению $u \in U_{a d}$. Заметим (см. [29, теорема 2]), что для любого $u \in U_{a d}$ функционал качества (4.1) корректно определен, так как $y(u) \in H^{1}(Q) \subset L^{2}(Q)$. Введем также обозначения

$$
y(0) \equiv y(y, 0), \quad p(0) \equiv p(x, 0), \quad y(T) \equiv y(x, T), \quad p(T) \equiv p(x, T) .
$$

Будем решать задачу оптимального управления (3.1)-(3.3), (4.2) как задачу оптимизации, в которой функция $u$ и начальные условия $y(0)$ неизвестны.

Легко показать, что оптимальное управление $u^{0}$, которое соответствует условию $y^{0}(0)$, задает наименьшее значение для функционала качества. Все другие управления $u^{0^{\prime}}$, которые соответствуют начальному условию $y(0) \in K$, задают большее значение для функционала качества. Продолжая таким образом и не имея дополнительной информации о множестве $K$ и функционале качества, можно достичь некоторого компромисса.

Используя теорему Дубовицкого-Милютина (см. [5, теорема 2.2]), выведем необходимые и достаточные условия оптимальности для задачи (3.1)-(3.3), (4.2). Идея метода ДубовицкогоМилютина детально описана в [5], и мы опускаем его описание.

Решение поставленной задачи оптимального управления эквивалентно поиску тройки $\left(y^{0}(0), y^{0}, u^{0}\right) \in E$, которая удовлетворяет уравнениям (3.1)-(3.3) и минимизирует функционал качества (4.1) с ограничениями на управление (4.2), где $K \times Y \times U=E=E_{1} \times E_{2} \times E_{3}$.

Сформулируем необходимые условия оптимальности для задачи (3.1)-(3.3) в следующей теореме оптимизации.

Теорема 4.1. Пусть $\left(y^{0}, u^{0}\right)$ - оптимальное решение задачи оптимального управления (3.1)(3.3), (4.1), (4.2). При выполнении указанных выше предположений существует $p \in W(0, T)$, удовлетворяющее (в слабом смысле) сопряженному уравнению, представленному ниже, и выполняется следующая система уравнений и неравенств в частных производных: 
(а) уравнение состояния:

$$
\begin{array}{ll}
\frac{\partial y^{0}}{\partial t}+\mathcal{A} y^{0}=u, & x \in \Omega, t \in(0, T), \\
\frac{\partial y^{0}(x, t)}{\partial \nu_{\mathcal{A}}}=0, & x \in \Gamma, t \in(0, T), \\
y^{0}(x, 0) \in K, & x \in \Omega ;
\end{array}
$$

(b) сопряженное уравнение:

$$
\begin{array}{cl}
-\frac{\partial p}{\partial t}+\mathcal{A}^{*}(t) p=\lambda_{1}\left(y^{0}-z_{d}\right), & x \in \Omega, t \in(0, T), \\
\frac{\partial p}{\partial \nu_{\mathcal{A}^{*}}}=0, & (x, t) \in Q, \\
p(x, T)=0, & x \in \Omega ;
\end{array}
$$

(с) условия максимума:

$$
\begin{array}{ll}
\int_{0}^{T} \int_{\Omega}\left(p+\lambda_{2} N u^{0}\right)\left(u-u^{0}\right) d x d t \geqslant 0 & \forall u \in U_{a d}, \\
\int_{\Omega} p(0)\left[k-y^{0}(0)\right] d x \geqslant 0 & \forall k \in K .
\end{array}
$$

Здесь

$$
\mathcal{A}^{*}(t) p=-\sum_{i, j=1}^{n} \frac{\partial}{\partial x_{j}}\left(a_{i j}(x, t) \frac{\partial p}{\partial x_{i}}\right)+a_{0}(x, t) p
$$

- сопряљенный оператор для $\mathcal{A}(t)$.

Доказательство. Согласно теореме Дубовицкого-Милютина 2.2 аппроксимируем множество, представляющее ограничения-неравенства, с помощью регулярного допустимого конуса, равенство ограничено регулярным касательным конусом, а функционал качества - регулярным конусом улучшения.

4.1. Анализ ограничений-равенств. Множество $Q_{1}$, представляющее ограничение-равенство, имеет вид

$$
Q_{1}:=\left\{(y(0), y, u) \in E ; \frac{\partial y}{\partial t}+\mathcal{A} y=u, x \in \Omega, t \in(0, T) ; \frac{\partial y}{\partial \nu_{\mathcal{A}}}=0, x \in \Gamma, t \in(0, T)\right\} .
$$

Построим регулярный касательный конус множества $Q_{1}$, используя теорему Люстерника (см. [1, теорема 9.1]). Для этой цели определим оператор

$$
P(y(0), y, u):=\left(\frac{\partial y}{\partial t}+\mathcal{A} y-u, \frac{\partial y}{\partial \nu_{\mathcal{A}}}\right) .
$$

Этот оператор является отображением из множества

$$
\mathcal{W}:=H^{1}(\Omega) \times L^{2}\left(0, T, H^{1}(\Omega)\right) \times L^{2}(Q)
$$

в множество

$$
\mathcal{Z}:=L^{2}\left(0, T, H^{-1}(\Omega)\right) \times L^{2}\left(0, T, H^{1}(\Omega)\right) .
$$

Производная Фреше оператора $P$ может быть записана в виде

$$
P^{\prime}\left(y^{0}(0), y^{0}, v^{0}\right)(\bar{y}(0), \bar{y}, \bar{v}):=\left(\frac{\partial \bar{y}}{\partial t}+\mathcal{A} \bar{y}-\bar{u}, \frac{\partial \bar{y}}{\partial \nu_{\mathcal{A}}}\right) .
$$

Действительно, $\partial / \partial t, A$ и $\partial / \partial \nu_{A}$ - линейные и ограниченные отображения (см. [7, теорема 2.1]). 
Используя [29, теорема 2], можно доказать, что $P^{\prime}\left(y^{0}(0), y^{0}, v^{0}\right)$ является биективным оператором из пространства $\mathcal{W}$ в пространство $\mathcal{Z}$.

Поскольку выполнены предположения теоремы Люстерника, можно описать регулярный касательный конус для множества $Q_{1}$ в точке $\left(y^{0}(0), y^{0}, u^{0}\right)$ следующим образом:

$$
R T C\left(Q_{1},\left(y^{0}(0), y^{0}, u^{0}\right)\right)=\left\{(\bar{y}(0), \bar{y}, \bar{u}) \in E ; P^{\prime}\left(y^{0}(0), y^{0}, u^{0}\right)(\bar{y}(0), \bar{y}, \bar{u})=0\right\} .
$$

Легко заметить, что он является подмножеством. Таким образом (см. [1, теорема 10.1])) известна форма функционала, принадлежащего сопряженному конусу:

$$
f_{1}(\bar{y}(0), \bar{y}, \bar{v})=0 \quad \forall(\bar{y}(0), \bar{y}, \bar{v}) \in R T C\left(Q_{1},\left(y^{0}(0), y^{0}, u^{0}\right)\right) .
$$

4.2. Анализ ограничений на управления. Множество $Q_{2}=K \times Y \times U_{a d}$, представляющее ограничения-неравенства, является замкнутым и выпуклым с непустой внутренностью в пространстве $E$.

Используя [1, теорема 10.5], найдем функционал, принадлежащий сопряженному регулярному конусу, т.е.

$$
f_{2}(\bar{y}(0), \bar{y}, \bar{v}) \in\left[R T C\left(Q_{2},\left(y^{0}(0), y^{0}, u^{0}\right)\right)\right]^{*} .
$$

Заметим, что если $E_{1}, E_{2}, E_{3}$ - три линейные топологические пространства, то сопряженное пространство для $E=E_{1} \times E_{2} \times E_{3}$ имеет вид

$$
E^{*}=\left\{f=\left(f_{1}, f_{2}, f_{3}\right), f_{1} \in E_{1}^{*}, f_{2} \in E_{2}^{*}, f_{3} \in E_{3}^{*}\right\}, \quad f(x)=f_{1}\left(x_{1}\right)+f_{2}\left(x_{2}\right)+f_{3}\left(x_{3}\right) .
$$

Таким образом, запишем функционал $f_{2}(\bar{y}(0), \bar{y}, \bar{v})$ следующим образом:

$$
f_{2}(\bar{y}(0), \bar{y}, \bar{u})=f_{1}^{1}(\bar{y})+f_{2}^{1}(\bar{y}(0))+f_{2}^{2}(\bar{u}),
$$

где

$$
f_{1}^{1}(\bar{y})=0 \quad \forall y \in Y
$$

(см. [1, теорема 10.1]), $f_{2}^{1}(\bar{y}(0)), f_{2}^{2}(\bar{v})$ - опорные функционалы для множеств $K$ и $U_{a d}$ в точках $y^{0}(0)$ и $u^{0}$ соответственно (см. [1, теорема 10.5]).

4.3. Анализ функиионала качества. Используя [1, теорема 7.5], найдем регулярный конус улучшения функционала качества (4.1) в виде

$$
R T C\left(I,\left(y^{0}(0), y^{0}, u^{0}\right)\right)=\left\{(\bar{y}(0), \bar{y}, \bar{u}) \in E ; I^{\prime}\left(y^{0}(0), y^{0}, u^{0}\right)(\bar{y}(0), \bar{y}, \bar{u})<0\right\},
$$

где $I^{\prime}\left(y^{0}(0), y^{0}, u^{0}\right)(\bar{y}(0), \bar{y}, \bar{u})$ - производная Фреше функционала качества (4.1), которая может быть выражена следующим образом:

$$
I^{\prime}\left(y^{0}(0), y^{0}, u^{0}\right)(\bar{y}(0), \bar{y}, \bar{u})=2 \lambda_{1} \int_{Q}\left(y^{0}-z_{d}\right) \bar{y} d x d t+2 \lambda_{2} \int_{Q}\left(N u^{0}\right) \bar{u} d x d t .
$$

На основе [1, теорема 10.2] найдем функционал, соответствующий сопряженному регулярному конусу улучшения, который имеет вид

$$
f_{3}(\bar{y}(0), \bar{y}, \bar{v})=-\lambda_{0} \lambda_{1} \int_{Q}\left(y^{0}-z_{d}\right) \bar{y} d x d t-\lambda_{0} \lambda_{2} \int_{Q}\left(N v^{0}\right) \bar{v} d \Gamma d t
$$

где $\lambda_{0}>0$. 
4.4. Анализ уравнения Эйлера-Лагранжа. Уравнение Эйлера-Лагранжа для нашей задачи оптимизации имеет вид

$$
\sum_{i=1}^{3} f_{i}=0
$$

Пусть $p(x, t)$ - решение задачи (4.7)-(4.9) для $u^{0}, y^{0}(0), y^{0}$ и обозначим через $\bar{y}$ решение для $P^{\prime}(\bar{y}(0), \bar{y}, \bar{u})=0$ при любых фиксированных $\bar{y}(0)$ и $\bar{u}$. Затем, принимая во внимание (4.17), (4.18) и (4.20), можно выразить (4.21) в виде

$$
\begin{aligned}
f_{2}^{2}(\bar{y}(0), \bar{y}, \bar{u})+f_{2}^{3}(\bar{u})=\lambda_{0} \lambda_{1} \int_{Q}\left(y^{0}-z_{d}\right) \bar{y} d x d t+\lambda_{0} \lambda_{2} \int_{Q}\left(N u^{0}\right) \bar{u} d \Gamma d t \\
\forall(\bar{y}(0), \bar{y}, \bar{u}) \in R T C\left(Q_{1},(\bar{y}(0), \bar{y}, \bar{u})\right) .
\end{aligned}
$$

Перенесем первую компоненту правой части уравнения (4.22), введя сопряженные переменные с помощью уравнения (4.7) и используя формулы (4.8), (4.9), (4.15) и (4.16). Получаем

$$
\begin{aligned}
\lambda_{0} \lambda_{1} \int_{Q}\left(y^{0}-z_{d}\right) \bar{y} d x d t=\lambda_{0} \int_{Q}\left(-\frac{\partial p}{\partial t}+\mathcal{A}^{*} p\right) \bar{y} d x d t & = \\
& =\lambda_{0} \int_{\Omega} p(0) \bar{y}(0) d x+\lambda_{0} \int_{Q} p \frac{\partial \bar{y}}{\partial t} d x d t+\lambda_{0} \int_{Q} \mathcal{A}^{*} p \bar{y} d x d t .
\end{aligned}
$$

Последнее слагаемое в (4.23) в силу формулы Грина может быть переписано в виде

$$
\lambda_{0} \int_{Q} \mathcal{A}^{*} p \bar{y} d x d t=\lambda_{0} \int_{Q} p \mathcal{A} \bar{y} d x d t+\lambda_{0} \int_{0}^{T} \int_{\Gamma} p \frac{\partial \bar{y}}{\partial \nu_{\mathcal{A}}} d \Gamma d t-\lambda_{0} \int_{0}^{T} \int_{\Gamma} \frac{\partial p}{\partial \nu_{\mathcal{A}^{*}}} \bar{y} d \Gamma d t .
$$

Воспользуемся граничными условиями (4.5) и (4.8); тогда обратятся в нуль второй и третий интеграл в правой части (4.24):

$$
\lambda_{0} \int_{Q} \mathcal{A}^{*} p \bar{y} d x d t=\lambda_{0} \int_{Q} p \mathcal{A} \bar{y} d x d t
$$

Подставляя (4.25) в (4.23), имеем:

$$
\begin{aligned}
\lambda_{0} \lambda_{1} \int_{Q}\left(y^{0}-z_{d}\right) \bar{y} d x d t & =\lambda_{0} \int_{\Omega} p(0) \bar{y}(0) d x+\lambda_{0} \int_{Q} p \frac{\partial \bar{y}}{\partial t} d x d t+\lambda_{0} \int_{Q} p \mathcal{A} \bar{y} d x d t= \\
& =\lambda_{0} \int_{\Omega} p(0) \bar{y}(0) d x+\lambda_{0} \int_{Q} p\left(\frac{\partial \bar{y}}{\partial t}+\mathcal{A} \bar{y}\right) d x d t= \\
& =\lambda_{0} \int_{\Omega} p(0) \bar{y}(0) d x+\lambda_{0} \int_{Q} p \bar{u} d x d t .
\end{aligned}
$$

Подставляя (4.26) в (4.22), получаем:

$$
f_{2}^{2}(\bar{y}(0), \bar{y}, \bar{u})+f_{2}^{3}(\bar{u})=\lambda_{0} \int_{\Omega} p(0) \bar{y}(0) d x+\lambda_{0} \int_{Q}\left(p(x, t)+\lambda_{2} N u^{0}\right) \bar{u} d x d t .
$$

Используя определение опорного функционала (см. [1]) и разделив в полученном неравенстве оба слагаемых на $\lambda_{0}$, получим

$$
\int_{\Omega} p(0)\left[k-y^{0}(0)\right] d x+\int_{Q}\left(p(x, t)+\lambda_{2} N u^{0}\right)\left(u-u^{0}\right) d x d t \geqslant 0
$$


для всех $k \in K$ и всех $u \in U_{a d}$. Последнее неравенство эквивалентно условию максимума (4.10), (4.11).

Чтобы доказать достаточность выведенных условий оптимальности, воспользуемся тем фактом, что ограничения и функционал качества выпуклы и существует такая точка $(\tilde{y}(0), \tilde{y}, \tilde{u}) \in$ $\operatorname{int} Q_{2}$, что $(\tilde{y}(0), \tilde{y}, \tilde{u}) \in Q_{1}$ (см. [29, теорема 2] и [1, теорема 15.2]). Этот факт следует напрямую из существования непустой внутренности множества $Q_{2}$ и существования решения уравнений (3.1)(3.3). Это замечание завершает доказательство теоремы 4.1.

Замечание 4.1. Можно рассмотреть аналогичную задачу управления с функционалом качества

$$
\hat{I}(y, u)=\lambda_{1} \int_{\Sigma}|y(u)|_{\Sigma}-\left.z_{\Sigma d}\right|^{2} d \Gamma d t+\lambda_{2} \int_{0}^{T} \int_{\Gamma}(N u) u d \Gamma d t
$$

где $z_{\Sigma d}$ - заданный элемент в $L^{2}(\Sigma)$. Из [29, теорема 2] и теоремы о следе (см. [7]) вытекает, что для каждого $u \in L^{2}(\Sigma)$ существует единственное решение $y \in W(0, T)$ с $\left.y\right|_{\Sigma} \in L^{2}(\Sigma)$. Тогда определено значение $\hat{I}(y, v)$.

Решение сформулированной задачи оптимального управления эквивалентно поиску тройки $\left(y^{0}(0), y^{0}, u^{0}\right) \in E=H^{1}(\Omega) \times W(0, T) \times L^{2}(\Sigma)$, которая удовлетворяет уравнениям (3.1)-(3.3) и минимизирует функционал качества (4.29) с ограничениями на управление (4.2). Можно доказать следующую теорему.

Теорема 4.2. Решение задачи оптимизачии (3.1)-(3.3), (4.29), (4.2) существует и единственно при выполнении допущений, упомянутых выше. Необходимые и достаточные условия оптимальности характеризуются следующей системой уравнений и неравенств в частных производных:

(i) уравнение состояния (4.3)-(4.6),

(ii) сопряженное уравнение

$$
\begin{array}{cl}
-\frac{\partial p}{\partial t}+\mathcal{A}^{*} p=0 & x \in \Omega, t \in(0, T), \\
\frac{\partial p}{\partial \nu_{\mathcal{A}^{*}}}=\lambda_{1}\left(\left.y^{0}\right|_{\Sigma}-z_{\Sigma d}\right), & (x, t) \in \Sigma, \\
p(x, T)=0, & x \in \Omega,
\end{array}
$$

(iii) условия максимума

$$
\begin{array}{ll}
\int_{0}^{T} \int_{\Gamma}\left(p+\lambda_{2} N u^{0}\right)\left(u-u^{0}\right) d \Gamma d t \geqslant 0 & \forall u \in U_{a d}, \\
\int_{\Omega} p(0)\left(k-y^{0}(0)\right) d x \geqslant 0 & \forall k \in K .
\end{array}
$$

Идея доказательства теоремы 4.2 аналогична той, что использовалась в доказательстве теоремы 4.1.

Следует отметить, что выведенные выше условия оптимальности (теоремы 4.1, 4.2) позволяют получить аналитическую формулу для оптимального управления лишь в частных случаях (например, когда нет ограничений на управления). Это вытекает из следующего: определение функции $p(x, t)$ в условии максимума возможно из сопряженного уравнения тогда и только тогда, когда известно, что $y^{0}(x, t)$ соответствует управлению $v^{0}(x, t)$. Эти взаимные связи затрудняют отдельное использование выведенной оптимизации. Таким образом, мы отказываемся от точного определения оптимального управления и используем приближенные методы. 
Замечание 4.2. Можно также рассмотреть аналогичную задачу управления с функционалом качества

$$
I(y, u)=\int_{Q} F(x, t, y, u) d x d t \rightarrow \min ,
$$

где $F: \mathbb{R}^{n} \times \mathbb{R}_{+}^{1} \times \mathbb{R}^{1} \times \mathbb{R}^{1} \rightarrow \mathbb{R}^{1}$ удовлетворяет следующим условиям:

$\left(A_{1}\right) F(x, t, y, u)$ непрерывно по $(x, t, y, u)$;

$\left(A_{2}\right)$ существуют $F_{y}(x, t, y, u), F_{u}(x, t, y, u)$, которые непрерывны по $(x, t, y, u)$,

$\left(A_{3}\right) F(x, t, y, u)$ строго выпукло по $(y, u)$, т.е.

$$
F\left(x, t, \lambda y_{1}+(1-\lambda) y_{2}, \lambda u_{1}+(1-\lambda) u_{2}\right)<\lambda F\left(x, t, y_{1}, u_{1}\right)+(1-\lambda) F\left(x, t, y_{2}, u_{2}\right)
$$

для всех $y_{1}, y_{2}, u_{1}, u_{2} \in \mathbb{R}^{n},\left(y_{1}, u_{1}\right) \neq\left(y_{2}, u_{2}\right), \lambda \in(0,1)$.

Из [29, теорема 2] и [6, теорема 2.1] вытекает что, для любого $u \in L^{2}(Q)$ существует единственное решение $y \in W(0, T)$. Тогда определена $I(y, u)$, заданная с помощью (4.35). Тогда решение сформулированной задачи оптимального управления эквивалентно поиску тройки $\left(y^{0}(0), y^{0}, u^{0}\right) \in E=H^{1}(\Omega) \times W(0, T) \times L^{2}(Q)$, удовлетворяющей уравнениям (3.1)-(3.3) и минимизирующей функционал качества (4.35) с ограничениями на управления (4.2). Можно доказать следующую теорему.

Теорема 4.3. Решение задачи оптимизации (3.1)-(3.3), (4.35), (4.2) существует и единственно при допущениях, упомянутых выше. Необходимые и достаточные условия характеризуются следующей системой уравнений и неравенств в частных производных:

(1) уравнение состояния (4.3)-(4.6),

(2) сопряженное уравнение

$$
\begin{array}{cl}
-\frac{\partial p}{\partial t}+\mathcal{A}^{*} p=F_{y}, & x \in \Omega, t \in(0, T), \\
\frac{\partial p(x, t)}{\partial \nu_{\mathcal{A}}}=0, & (x, t) \in \Sigma, \\
p(x, T)=0, & x \in \Omega ;
\end{array}
$$

(3) условия максимума

$$
\begin{array}{ll}
\int_{0}^{T} \int_{\Omega}\left(p+F_{u}\right)\left(u-u^{0}\right) d x d t \geqslant 0 & \forall u \in U_{a d}, \\
\int_{\Omega} p(0)\left(k-y^{0}(0)\right) d x \geqslant 0 & \forall k \in K,
\end{array}
$$

где индекс «0» обозначает оптимальный элемент, а $F_{y}, F_{u}$ - производные Фреше функиии $F$ относительно $у$, и в точке $\left(y^{0}, y^{0}\right)$.

Идея доказательства теоремы 4.3 аналогична идее доказательства теоремы 4.1.

5. Заключение. Задача оптимизации, представленная в работе, представляет собой обобщение задачи оптимального граничного управления для параболических систем с граничными условиями второго порядка, рассмотренной в [23-25], в случае параболических систем с неполной информацией о начальных условиях. Можно рассмотреть задачу оптимального управления для параболических граничных условий Дирихле с неполной информацией о начальных условиях.

Также очевидно, что множество вариаций описанных выше задач можно исследовать с помощью формализма Дубовицкого-Милютина (см. [6, 8-22]), модифицируя

(а) граничные условия (Дирихле, второго порядка, смешанные и т.д.);

(b) природу управления (распределенное, ограниченное и т.д.);

(c) тип наблюдения (распределенное, ограниченное и т.д.);

(d) исходную дифференциальную систему; 
(е) временные запаздывания (постоянные временные запаздывания, изменяющиеся во времени запаздывания, многократные изменяющиеся во времени запаздывания, заданные в интегральной форме временные запаздывания и т.д.);

(f) число переменных (конечное число переменных, бесконечное число переменных и т.д.);

(g) тип уравнения (эллиптическое, параболическое, гиперболическое и т.д.);

(h) порядок уравнения (второй порядок, бесконечный, дробный порядок и т.д.; см. [14-17]);

(i) тип управления (задача оптимального управления, задача быстродействия и т.д.).

Эти задачи нуждаются в дальнейшем изучении будут рассмотрены авторами позже.

\section{СПИСОК ЛИТЕРАТУРЫ}

1. Гирсанов И. В. Лекции по математической теории экстремальных задач. - М.: Изд-во МГУ, 1970.

2. Дубинский Ю. А. Пространства Соболева бесконечного порядка и поведение решений некоторых краевых задач при неограниченном возрастании порядка уравнения / Мат. сб. - 1975. — 98 (140), № 2 (10). - C. $163-184$.

3. Дубинский Ю. А. Нетривиальность пространств Соболева бесконечного порядка в случае полного эвклидова пространства и тора// Мат. сб. - 1976. - 100 (142), № 3 (7). - С. 436-446.

4. Дубовицкий A. Я., Милютин А. А. Задачи на экстремум при наличии ограничений// Докл. АН CCCP. - 1963. - 149, № 4. - С. 759-762.

5. Дубовичкий А. Я., Милютин А. А. Задачи на экстремум при наличии ограничений// Ж. вычисл. мат. мат. физ. - 1965. - 5, № 3. - С. 395-453.

6. Лионс Ж.-Л. Оптимальное управление системами, описываемыми уравнениями в частных производных. - М.: Мир, 1972.

7. Лионс Ж.-Л., Мадженес Э. Неоднородные граничные задачи и их приложения. — М.: Мир, 1971.

8. Bahaa G. M. Quadratic Pareto optimal control of parabolic equation with state-control constraints and an infinite number of variables// IMA J. Math. Control Inform. — 2003. — 20. — P. 167-178.

9. Bahaa G. M. Time-optimal control problem for parabolic equations with control constraints and infinite number of variables// IMA J. Math. Control Inform. - 2005. - 22. - P. 364-375.

10. Bahaa G. M. Optimal control for cooperative parabolic systems governed by Schrödinger operator with control constraints// IMA J. Math. Control Inform. - 2007. - 24. - P. 1-12.

11. Bahaa G. M. Optimal control problems of parabolic equations with an infinite number of variables and with equality constraints// IMA J. Math. Control Inform. - 2008. - 25. - P. 37-48.

12. Bahaa G. M. Boundary control problem of infinite order distributed hyperbolic systems involving time lags// Intel. Control Automat. - 2012. - 3, № 3. - P. 211-221.

13. Bahaa G. M. Optimality conditions for infinite order distributed parabolic systems with multiple time delays given in integral form// J. Appl. Math. - 2012. - 2012. - P. 1-25.

14. Bahaa G. M. Fractional optimal control problem for variational inequalities with control constraints// IMA J. Math. Control Inform. - 2016. - 33, № 3. - P. 1-16.

15. Bahaa G. M. Fractional optimal control problem for differential system with control constraints// Filomat. - 2016. - 30, № 8. - P. 2177-2189.

16. Bahaa G. M. Fractional optimal control problem for infinite order system with control constraints// Adv. Differ. Equations. - 2016. - 250.

17. Bahaa G. M. Fractional optimal control problem for differential system with delay argument// Adv. Differ. Equations. - 2017. - 69 .

18. Bahaa G. M., Kotarski W. Optimality conditions for $n \times n$ infinite order parabolic coupled systems with control constraints and general performance index// IMA J. Math. Control Inform. - 2008. — 25. P. 49-57.

19. Bahaa G. M., Kotarski W. Time-optimal control of infinite order distributed parabolic systems involving multiple time-varying lags// Numer. Funct. Anal. Optim. — 2016. — № 9. — P. 1066-1088.

20. Bahaa G. M. and Tharwat M. M. Optimal control problem for infinite variables hyperbolic systems with time lags// Arch. Control Sci. — 2011. — 21, № 4. - P. 373-393.

21. Bahaa G. M. and Tharwat M. M. Time-optimal control of infinite order parabolic system with time lags given in integral form// J. Inform. Optim. Sci. - 2012. - 33, № 2-3. - P. 233-258.

22. Bahaa G. M. and Tharwat M. M. Optimal boundary control for infinite variables parabolic systems with time lags given in integral form// Iran. J. Sci. Technol. — 2012. — A3. — P. 277-291. 
23. Kotarski $W$. Some problems of optimal and Pareto optimal control for distributed parameter systems// Rep. Silesian Univ. - 1997. — № 1668. - P. 1-93.

24. Kotarski W., El-Saify H. A., Bahaa G. M. Optimal control of parabolic equation with an infinite number of variables for non-standard functional and time delay// IMA J. Math. Control Inform. — 2002. — 19, № 4. - P. 461-476.

25. Kowalewski A., Kotarski W. On application of Milutin-Dubovicki's theorem to an optimal control problem for systems described by partial differential equations of hyperbolic type with time delay// Systems Sci. 1981. — 7, № 1. - P. 55-74.

26. Tröltzsch F. Optimality conditions for parabolic control problems and applications. - Leipzig: Teubner, 1984.

27. Walczak S. One some control problems// Acta Univ. Lodz. Folia Math. — 1984. - 1. - P. 187-196.

28. Walczak $S$. Some properties of cones in normed spaces and their application investigating extremal problems// J. Optim. Theory Appl. — 1984. — 42, № 2. — P. 561-582.

29. Wang P. K. C. Optimal control of parabolic systems with boundary conditions involving time delay// SIAM J. Control. — 1975. - 13. - P. 274-293.

Bahaa G. M.

Университет Тайба, Эль-Мадина-эль-Мунаввара, Саудовская Аравия;

Университет Бени-Суэйф, Бени-Суэйф, Египет

E-mail: Bahaa_gm@yahoo.com 